\title{
THE VECTORCARDIOGRAM OF CARDIAC INFARCTION
}

\author{
BY \\ WALLACE BRIGDEN, AND JOHN SHILLINGFORD
}

From the Cardiac Department of the London Hospital

Received December 18, 1951

A simple method of drawing the three-plane projection of the spatial vectorcardiogram has been described (Shillingford and Brigden, 1951). A series of normal vectorcardiograms were studied and found to have certain constant characteristics that were comparable with the results obtained by electronic methods. In this paper we report the vectorcardiographic findings of extensive cardiac infarction, and the abnormal patterns obtained have been related to the QRS-T complexes recorded from the same patients in the conventional manner. Duchosal and Sulzer (1949) and Vastesaeger (1949) have studied the vectorcardiogram of cardiac infarction, using an electronic apparatus. Our findings are in substantial agreement with those recently reported by Scherlis and Grishman (1951); their three-plane vectorcardiograms were seen simultaneously on a tribeam cathode-ray tube and photographed on one film.

\section{METHOD}

The method of integrating the scalar electrocardiograms has been the same as we described, but the lead positions of the reference system have been slightly modified. The voltage obtained from leads in the horizontal plane in the system advocated by Duchosal and Sulzer (1949) is small, and it was found that the higher voltage that could be obtained by bringing the electrodes nearer to the heart, made the vectorcardiogram easier to correlate with the conventional chest leads. The horizontal lead positions have therefore been raised from the iliac spine level to a plane through the xiphisternum; the reference system then becomes almost cuboidal. It is appreciated that there are certain theoretical objections to placing electrodes any nearer the heart, but this new position is considered to be a reasonable compromise that sacrifices little fundamental accuracy and facilitates interpretation of the loop. Grishman et al. (1951) have made similar changes in the lead positions.

The validity of this method is shown by the similarities of the QRS complexes reconstructed from our vectorcardiograms and the actual leads obtained by the conventional method. In Fig. 1A the reconstructed QRS complexes from the frontal plane loop are compared with the standard leads recorded from the same normal subject. A reconstruction of the unipolar chest leads from the horizontal plane loop is shown in Fig. 2A together with the recorded unipolar chest leads. The projections in three planes of the spatial vectorcardiogram have been drawn in 20 cases of cardiac infarction, of which 10 were anterior and 10 posterior in position. All observations were made with the subject in a reclining position. Each patient had a history of prolonged chest pain at rest, and an electrocardiogram that showed pathological Q waves.

\section{RESULTS}

Four examples of the vectorcardiogram in patients with posterior cardiac infarction are shown in Fig. 3, and a frontal projection is shown with the reconstructed complexes alongside the recorded limb leads in Fig. 1B. All the patients with this condition showed loops that were initially directed upwards in the frontal plane and tended to lie in the upper quadrants; the sagittal view (Fig. 3) of the loop shows the same upwards deviation. The horizontal projection showed no constant 


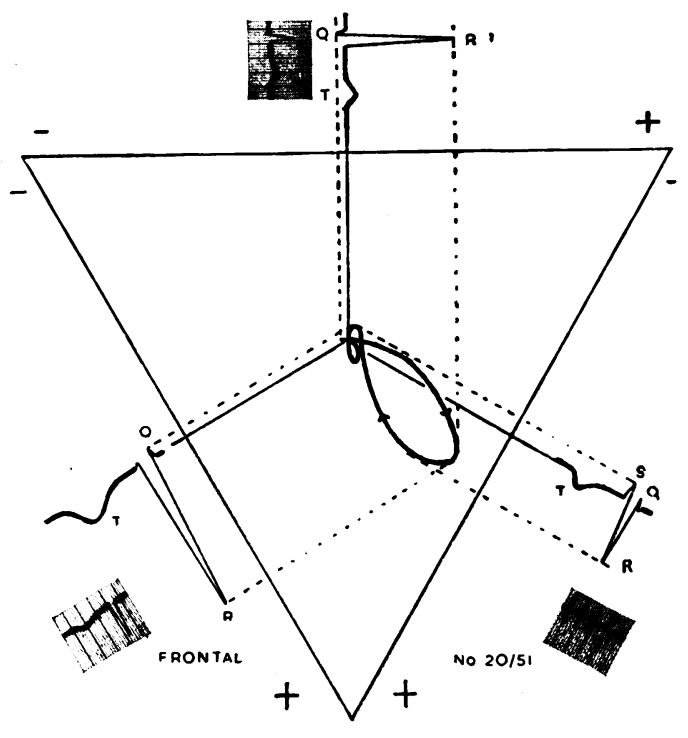

A

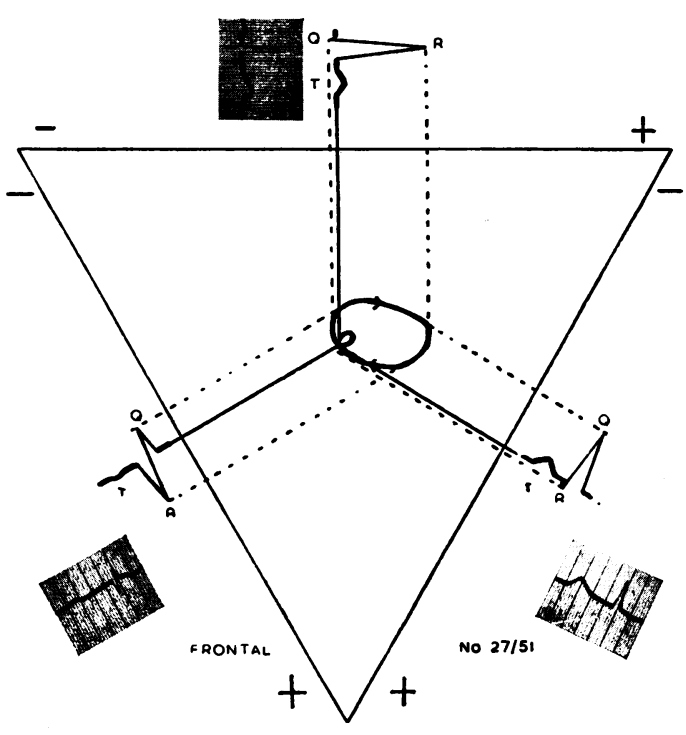

B

FIG. 1.-(A) The frontal projection of a normal vectorcardiogram with reconstruction of leads I, II, and III, and with actual recordings for comparison. (B) The frontal plane vectorcardiogram of a case of posterior cardiac infarction. A deep QII and QIII are associated with the upward shift of this loop.

abnormality, but in one case (Fig. 3, No. 1) there was a forward shift of the whole loop and in another (Fig. 3, No. 2) there was a forward indentation of the returning limb with a corresponding forward bulge of the outgoing limb. These abnormalities are believed to be due to an area of posterolateral infarction in addition to the area of inferior infarction. It will be seen (Fig. 1B) that this abnormal deviation of the cardiac vectors into the upper quadrants results in the appearance of Q waves in the reconstruction of leads II and III. The time taken for the inscription of the QRS loop was within normal limits, while its direction of rotation was not constant. The T loop was found to be directed upwards in the frontal plane, and there was little change in the horizontal plane.

Four examples of the vectorcardiogram in patients with anterior cardiac infarction are shown in Fig. 4, and the horizontal loop from one patient is shown in Fig. 2B with reconstructed chest leads alongside the unipolar chest leads 1, 4, and 7. In this group the QRS loop has shifted backwards in the chest, a change that can be seen both in the horizontal and sagittal projections. In the sagittal view neither the outgoing nor returning limb of the loop projects into the anterior quadrants, i.e. to the right of the vertical isoelectric line. This backwards shift of the QRS vectors results in the appearance of $Q$ waves in reconstructed anterior chest leads. The absence of any vectors in front of the isoelectric line in the sagittal view similarly is a reflection of the $Q$ waves found in the centre of the chest in anterior cardiac infarction. The time taken for the inscription of the QRS was within normal limits and the direction of the rotation of the loop was not constant. The T loop was found to be directed backwards and to the left, i.e. in the same direction as the QRS loop, but in the case of an old cardiac infarction the T loop was orientated in the normal way (Fig. 4, No. 2, 3, and 4).

It has been shown that when due allowances are made for the distance of the electrodes from the centre of the heart, tracings are obtained at opposite points on the surface of the chest that are mirror images (Duchosal, 1951). It was expected, therefore, that the retreat of the vector loop from the site of the infarction would result in the appearance of $R$ waves in leads taken diametrically opposite to those showing $\mathrm{Q}$ waves. This effect is well shown in a patient with anterior cardiac 


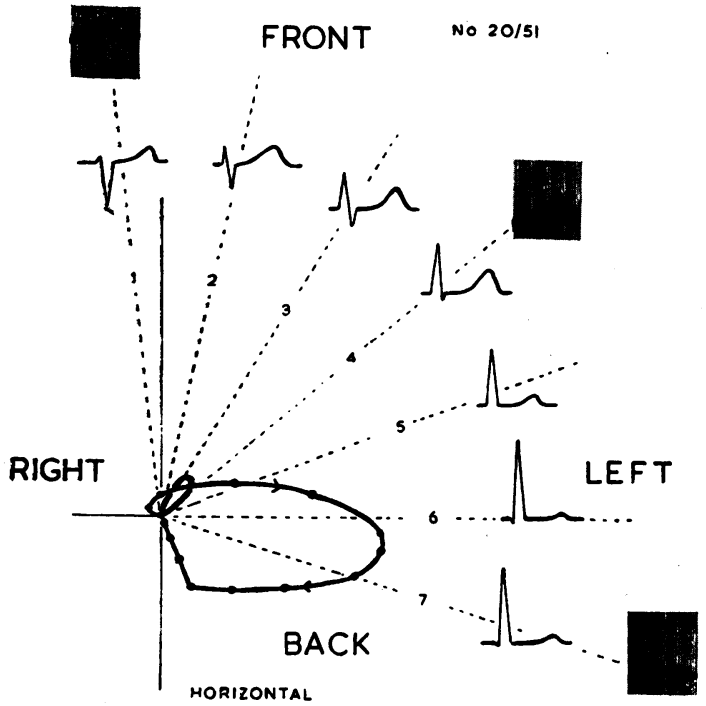

A

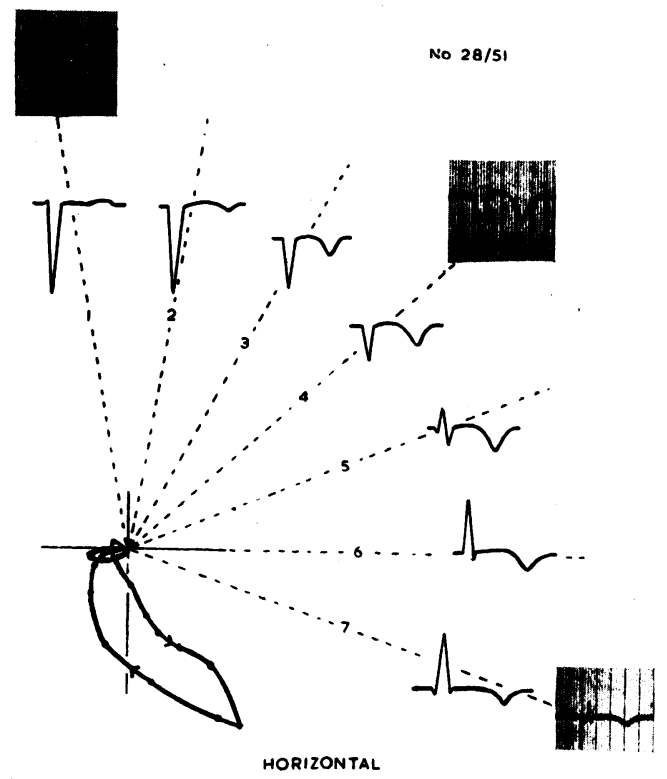

B

Fig. 2.-(A) The horizontal projection of a normal vectorcardiogram showing reconstructed chest leads and leads V1, V4, and V7. (B) A similar projection in anterior cardiac infarction. Deep " $Q$ waves" appear in the reconstructed QRS complexes due to backward shift of the loop. The leads V1, V4, and $\mathbf{V} 7$ are in the appropriate positions.

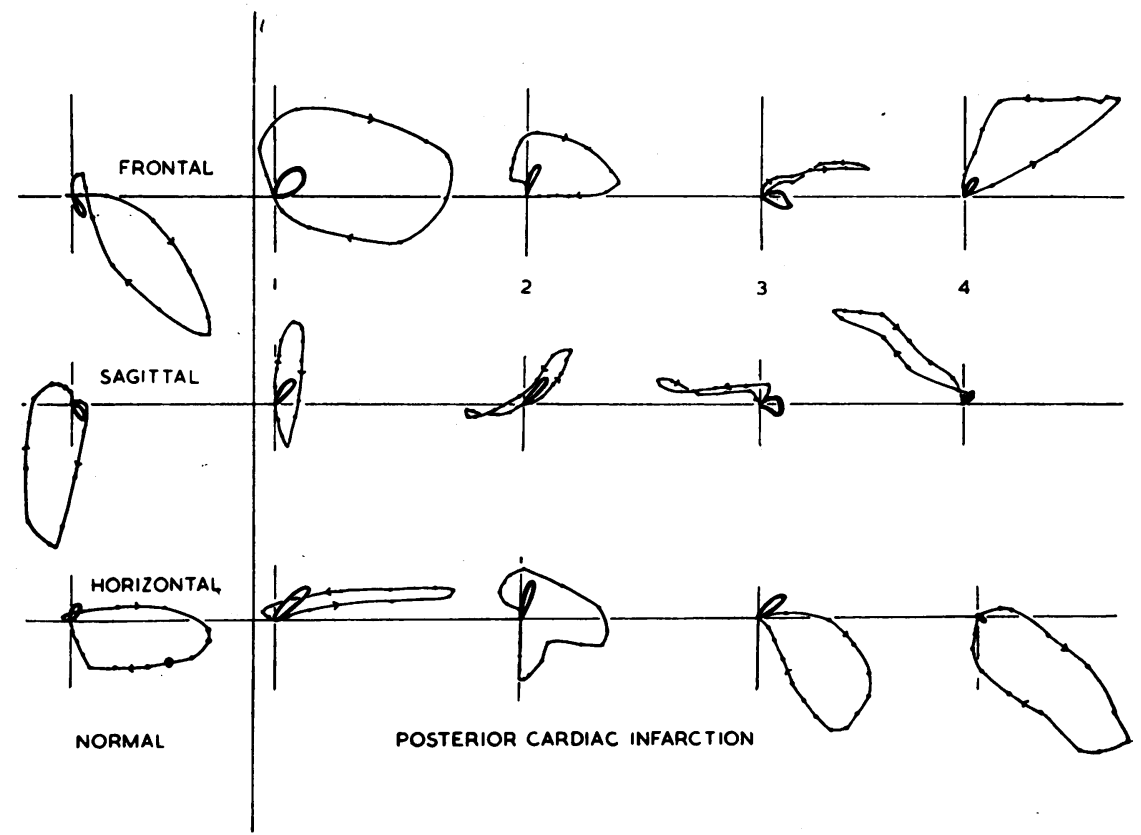

Fig. 3.-The frontal, sagittal, and horizontal vectorcardiograms of four patients with posterior cardiac infarction, for comparison with the normal. 


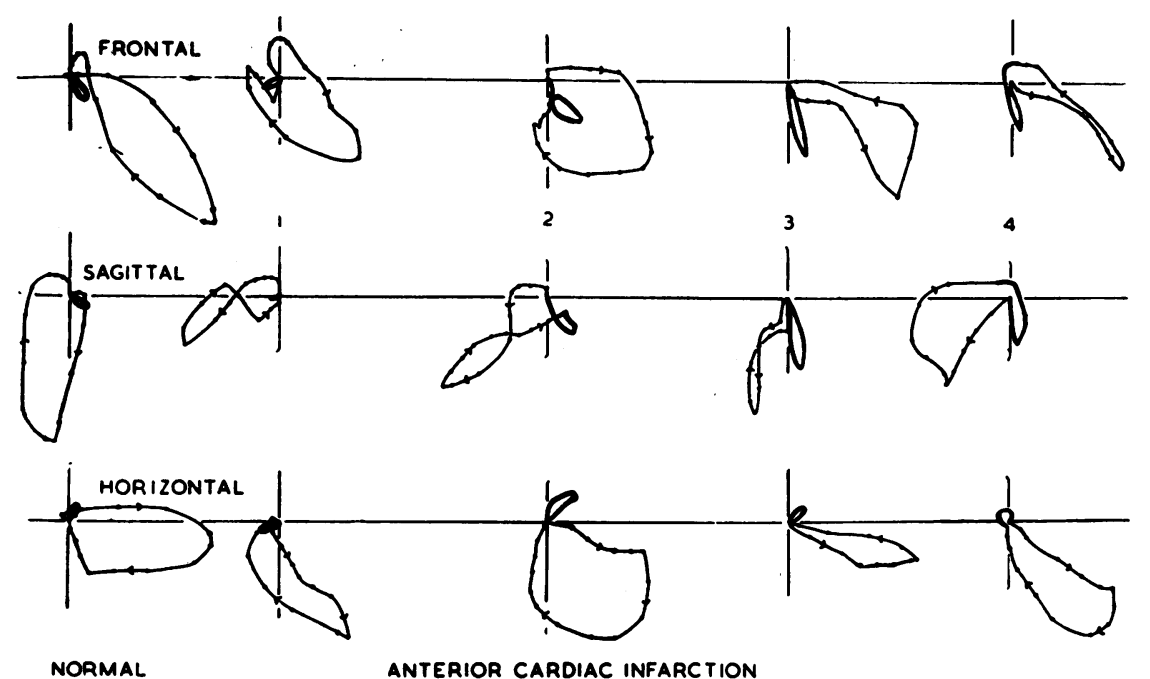

FIG. 4.-The frontal, sagittal, and horizontal vectorcardiograms of four patients with anterior cardiac infarction.

infarction on whom 12 unipolar leads were taken round the chest and correlated with the horizontal projection of the vectorcardiogram (Fig. 5).

\section{Discussion}

All the patients chosen for this study had evidence of cardiac infarction with pathological $Q$ waves in the electrocardiogram. The vectorcardiogram of all showed a characteristic shift of the loop away from the unipolar leads recording $Q$ waves. This is readily seen in the horizontal loop of anterior cardiac infarction as is shown in Fig. 2B. The reorientation of the loop away from the lead positions that show $Q$ waves is also seen in the case of posterior infarction for here the relevant unipolar lead with. $Q$ waves is VF, the axis of which is a line approximately bisecting the apex of Einthoven's triangle, and the loop in this case has moved away from the apex towards the base of the triangle.

It has frequently been shown in studies relating the electrocardiogram to morbid anatomy that $Q$ waves are found in leads anatomically related to the site of infarction. It therefore follows that the loop of cardiac vectors retreats from the area of infarction; thus, in anterior lesions it shifts backwards in the chest, and in posterior (or better inferior) myocardial infarction, the loop shifts in a cephalad direction. This retreat from the infarcted area (i.e. from leads showing $Q$ waves) inevitably results in the appearance of $\mathbf{R}$ waves in leads situated at opposite points on the surface. In cases of anterior infarction, therefore, $R$ waves are found at the back of the chest, although such positive waves are of small voltage owing to the increased distance of the electrodes from the heart. In cases of posterior infarction, this shift of the positive vectors into the upper quadrants results in the appearance of positivity in the basal unipolar leads which may be seen in the frequent occurrence of a primary $R$ wave in VR. The appearance of a prominent $R$ wave in lead V1 in cases of lateral myocardial infarction as described by Levy et al. (1950) is a similar manifestation of the preponderance of cardiac vectors away from this site of infarction. This change is well shown in the horizontal loop of Fig. 3, No. 2, where the forward bulge of the loop would produce a prominent $\mathbf{R}$ in $\mathrm{V} 1$.

This constant shift of cardiac vectors away from the damaged area is presumably caused by the preponderant effect of the remaining normal muscle. The $Q$ wave may be seen to represent a recession of the QRS loop away from the site of infarction rather than a transmission of negativity 


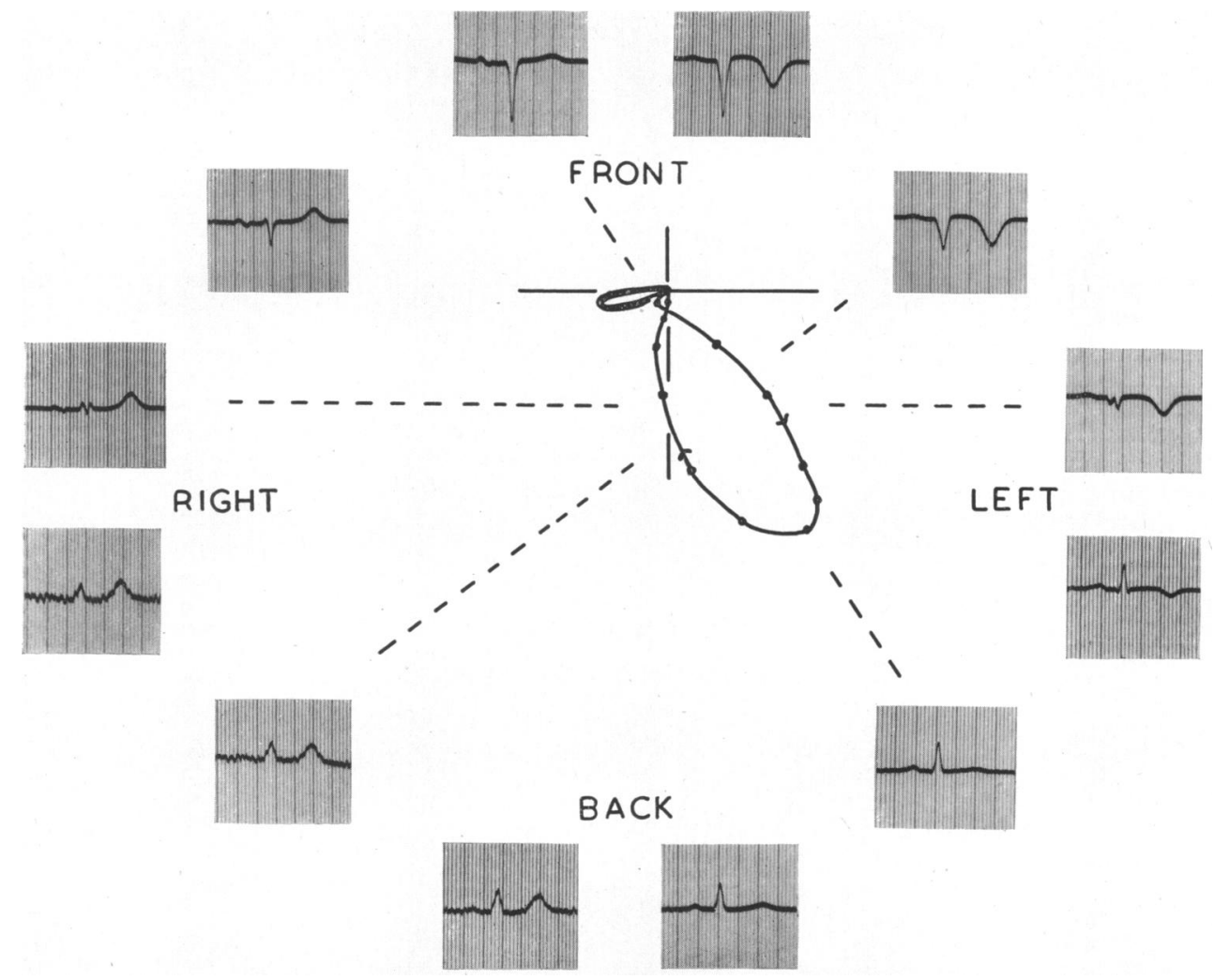

Fig. 5.-The horizontal projection of a vectorcardiogram of anterior cardiac infarction. The loop is characteristically deviated backwards with resultant $Q$ waves in conventional chest lead positions V1, V2, V4, and V6. QRS complex in leads at the back of chest show R waves and upright T waves. The mirror image effect may be observed in most pairs of leads.

from the cavity. This difference in concept depends rather on the investigator's attitude to the subject than a fundamental biophysical difference. However, it may be seen that the vector loop directs the attention to the extent and site of normal muscle as well as to the infarcted area of myocardium.

The changes in the T loop are similar to those of the QRS but the usual " recovery of T waves" is seen in some cases in the return of the $T$ loop to its normal orientation in the chest.

It is not suggested that the methods described add anything to the diagnosis of cardiac infarction for the site and severity of this condition can be assessed by clinical and the more orthodox electrocardiographic methods, but a knowledge of the vectorcardiogram in gross infarction provides a more unified picture of the abnormal electrical activity of the heart as a whole.

\section{SUMMARY}

Theoretical QRS-T complexes have been reconstructed from the frontal and horizontal plane projections of the spatial vectorcardiogram and shown to resemble closely those obtained by conventional electrocardiography. Three plane projections of the vectorcardiogram have been drawn in 20 cases of cardiac infarction.

The vectorcardiogram of anterior cardiac infarction shows a constant deviation of the loop from the site of infarction: this is best seen in the horizontal plane. A similar deviation of the 
loop away from the site of infarction is seen in cases of posterior infarction, and is shown best in the frontal plane.

The significance of these findings has been discussed in relation to the appearance of pathological waves in the conventional surface leads.

\section{REFERENCES}

Duchosal, P., and Sulzer, R. (1949). La Vectorcardiographie. S. Karger.

(1951). St. Cyres Lecture, National Heart Hospital, London.

Grishman, A., Borun, R., and Jaffe, H. (1951). Amer. Heart J., 41, 483.

Levy, L., Jacobs, H. J., Chestant, H. P., and Strauss, A. B. (1950). Amer. Heart J., 40, 447.

Scherlis, L., and Grishman, A. (1951). Amer. Heart J., 42, 24.

Shillingford, J., and Brigden, W. (1951). Brit. Heart J., 13, 233.

Vastesaeger, M. (1949). Acta Cardiol., 4, 22. 